REVISTA ECONOMÍA

Vol. 70, N. ${ }^{\circ}$ II2 (noviembre 20I8), I37-I48

\title{
LA CONSISTENCIA ACERVO-FLUJO, LA COHERENCIA MATRICIAL DEL VALOR Y LOS ESQUEMAS DE REPRODUCCIÓN SIMPLE DE K. MARX
}

\author{
PEDRO PÁEZ PÉREZ \\ PUCE, Instituto de Investigaciones Económicas \\ Recepción de manuscrito: 17 de septiembre de 2018 \\ Aceptación versión final: 26 de octubre de 2018
}

\begin{abstract}
Resumen Aplicando la metodología de Consistencia Acervo-Flujo (SFC), se exploran los más sencillos esquemas de reproducción de Marx — reproducción simple, 2 sectores-con una lectura diferente que permite derivar la emergencia de procesos complejos sobredeterminando las intencionalidades micro exante en los resultados macro expost, a partir, fundamentalmente, de identidades contables. La operación matricial de la Ley del Valor a través del fetiche de los precios de producción constriñe sistémicamente la maximización de la ganancia con una serie de externalidades que escapan a la voluntad - y a la percepción- de capitalistas y trabajadores individuales.
\end{abstract}

PALABRAS ClAVE K. Marx, crisis, esquemas de reproducción, economía poskeynesiana.

ABSTRACT From a Stock-Flow Consistency Framework, a new exploration into the simplest of Marx's Reproduction Schemes (2-Sector, Simple Reproduction) tracks the emergency of complex processes overruling the ex-ante micro intentions with ex-post macro results. The matrixlike operation of the price of production's fetishism as a transformation of value determinations, systemically restricts the profit-maximization drive with a set of externalities beyond the will and the perception of capitalists and workers.

KEYWORDS K. Marx, crisis, reproduction schemes, post-Keynesian economics.

JEL CODES B5, E11, E12.

\section{INTRODUCCIÓN}

Desde una lectura distinta, aquí se busca reflexionar y desarrollar las exploraciones muy originales de K. Marx ${ }^{1}$ en torno a los esquemas de reproducción simple que quedaron solamente en borrador, en gran parte solo como ilustraciones. Con el marco de referencia del álgebra lineal y la Consistencia Acervo-Flujo (Stock-Flow Consistency) que Marx anticipa y que solo empieza a tener un tratamiento sistemático en la literatura económica moderna, en particular entre las corrientes poskeynesianas, ${ }^{2}$ se encuentran conexiones que nos permiten entender la emergencia de fenómenos sistémicos ajenos a la voluntad - y posiblemente, la percepciónde los actores involucrados, incluso los más poderosos. 
Se estudia la operación matricial de la Ley del Valor a través del fetiche de los precios de producción y desde allí, las condiciones emergentes en la dinámica de los mercados capitalistas debidas a la correspondencia del balance en valor de uso y en valor de cambio de las complementariedades macroeconómicas entre sectores de la economía, ${ }^{3}$ de manera consistente en acervos y flujos y las determinaciones que esto implica en la dinámica general de la acumulación capitalista estructuralmente diferenciada.

Contra todo reduccionismo metodológico y evitando ecuaciones de comportamiento al máximo, se derivan, desde sencillas identidades contables, los efectos macroscópicos complejos que emergen - similares a los de la Ley de Gases sobre las moléculas- que condicionan las dinámicas diferenciales de las tasas de ganancia y acumulación.

\section{DETERMINACIONES EMERGENTES EN LOS BALANCES \\ INTERSECTORIALES Y REALIZACIÓN DE LAS GANANCIAS}

K. Marx busca el más sencillo paso para avanzar desde el análisis del volumen I de El capital en el cual se trata al capital como una relación de producción abstracta, contraponiendo a dueños de medios de producción con trabajadores que venden su fuerza de trabajo por un salario. Inicia el análisis avanzando en lo que sería el volumen ir asumiendo una economía totalmente capitalista, cerrada, de reproducción simple donde todo el capital constante se consume dentro del período de análisis y sin crédito ${ }^{4}$ y empieza su consideración de la agregación plural en el balance agregado del valor de cambio con los mínimos dos sectores caracterizados por tipo de valor de uso.

El capital individual contrata fuerza de trabajo por una masa salarial equivalente al capital variable (v) - en tanto genera un plus de valor (s) - que entra en acto de producir con los medios de producción comprados por un equivalente de capital que se mantendrá constante (c). El capital adelantado individual (d) busca en el mercado un reconocimiento proporcional de una tasa de ganancia ( $\mathrm{g}$ ) de ese plus de valor, que se espera resulte en un monto de capital individual (d') mayor al inicialmente adelantado.

No obstante, las condiciones de realización agregadas expost de las variables individuales no coinciden necesariamente con las expectativas, decisiones y propósitos individuales exante. ${ }^{5}$ La determinación «baja» del todo a las partes, del balance en agregados en valor de uso y valor de cambio que recuperan conflictivamente la unidad orgánica entre producción y consumo:
1) $C \equiv \sum i c i$
2) $V \equiv \sum i v i$
3) $S \equiv \sum i s i$
4) $P \equiv \sum i p i$
5) $D \equiv \sum i d i$

Estos agregados necesariamente deben corresponder en valor de cambio, pero los balances entre lo que se compra y lo que se vende no arroja un equilibrio neoclásico: el resultado expost incluye desempleo, desperdicio y mercancías no vendidas. El capital constante efectivamente utilizado en el agregado dentro del período analizado (C); el capital variable con una 
tasa salarial a la que efectivamente se contrata por debajo del pleno empleo (V) definiendo un ejército industrial de reserva; y un monto de plusvalor efectivamente realizado (S), igualan solamente el valor del producto total efectivamente vendido $(\mathrm{P})$ :

6) $\mathrm{C}+\mathrm{V}+\mathrm{S} \equiv \mathrm{P}$

Así, la tasa de explotación $\mathrm{r} \equiv \mathrm{s} / \mathrm{v}$ y la composición orgánica del capital, $\mathrm{o} \equiv \mathrm{c} / \mathrm{v}$ individuales que integran los procesos de decisión exante, resultan en relaciones expost que reflejan la emergencia de fenómenos que aparecen misteriosamente operados por el fetiche del mercado, pero que expresan la posibilidad general de crisis inherente en la mediación mercantil ${ }^{6}$ y exacerbada por la ley de movimiento sistémica fundamental de retirar del mercado un valor mayor que el inicialmente inyectado, maximizando las ganancias:

7) $\max \left(D^{\prime}-D\right)=\sum i \max \left(\mathrm{d}^{\prime} i-\mathrm{d} i\right)$

La operación de la ley del valor a través de los precios de producción irrumpe problemáticamente en tanto no hay garantía de realización de la ganancia. Y esa incertidumbre es sectorial y secuencialmente diferenciada por la brecha en tiempo y espacio entre capacidades de compra. ${ }^{7}$ El movimiento del capital D - M - D' queda representado en el agregado por:

8) $\mathrm{D} \equiv \mathrm{C}+\mathrm{V}$

9) $\mathrm{D}^{\prime} \equiv \mathrm{C}+\mathrm{V}+\mathrm{S}$

El total de las ganancias $(\mathrm{G})$ debe ser igual al plusvalor generado por los trabajadores, pero en proporción al capital adelantado. ${ }^{8}$

10) $\mathrm{D}^{\prime}-\mathrm{D}=\mathrm{S}=\mathrm{G}$

11) $\mathrm{D}^{\prime}=\mathrm{D}(1+\mathrm{g})$

La tasa de ganancia, g, puede ser expresada como una relación entre la tasa de plusvalor y la composición orgánica del capital:

12) $g \equiv \frac{s}{(c+v)} \equiv \frac{r}{(o+1)}$

Una división en sectores que agregan entre sí todas las actividades productivas, mutuamente excluyentes y aditivamente exhaustivas conforme el ejercicio de Marx permite entender la correspondencia necesaria entre los flujos agregados de energía y materiales en el plano ecológico de la autopoiesis sustancial de la vida del sujeto-especie y el plano del intercambio de equivalentes.

Es decir, la metodología de Consistencia Acervo-Flujo que Marx anticipa permite rastrear las restricciones que las identidades contables ineludibles impondrán compulsiva, pero opacamente sobre las transacciones indispensables y las motivaciones de los agentes individuales operando dentro de cada sector. La tabla (ver Tabla 1) caracteriza la matriz de los balances entre 
fuentes y usos que definen las proporciones en valor de cambio en los agregados del producto social y la demanda social. Este balance refleja la disciplina y proporcionalidad del trabajo socialmente necesario para la reproducción de los sectores, en la medida en que la masa de fuerza de trabajo distribuida en cada sector por su respectiva tasa salarial define el monto específico de capital variable (V), el mismo que escalado por la tasa de plusvalor (r) y la composición orgánica de capital (o), construye las columnas restantes, conforme el ejercicio inicial de Marx. ${ }^{9}$

La división social del trabajo exige una congruencia dinámica entre un sector I: producción de medios de producción; y un sector II: producción de medios de consumo. ${ }^{10}$ La totalidad del trabajo pasado o muerto cristalizado en los medios de producción queda representado por el vector columna C. Su peso en la evolución de los procesos de trabajo crece en el plano del metabolismo del sujeto-especie, asume, en el plano del valor de cambio, un monto que no necesariamente crece monotónicamente debido a que los propios cambios tecnológicos que determinan su crecimiento en tanto valores de uso presionan a su abaratamiento.

La proporción de medios de producción repartida en cada sector define una trayectoria dependiente en el tiempo y desde la perspectiva de la Consistencia Acervo-Flujo queda rigurosamente sobredeterminada por el costo hundido del capital fijo y por los coeficientes técnicos del proceso de trabajo que se expresan en los requerimientos de insumo en capital constante circulante - materias primas, energía y otros elementos de las condiciones generales del proceso de trabajo- que una tecnología dada plantea en proporción al capital constante fijo. Esas determinaciones por el lado de los usos, que tienen una vinculación con la composición orgánica de capital como veremos, exigen una correspondencia por el lado de las fuentes y define una proporción técnica entre el capital constante requerido por cada sector, en valor de uso y valor de cambio, esta última dada por: ${ }^{11}$

13) $\mathrm{j} \equiv \mathrm{c} 2 / \mathrm{c} 1$

Similarmente, la dinámica de la acumulación del capital debe cumplir en cada etapa un requerimiento agregado, tanto a nivel global como sectorial de la utilización de trabajo vivo sobre la base de la compra de fuerza de trabajo. Las condiciones técnicas y de lucha social define una proporción (h) entre el trabajo vivo movilizado para la producción de medios de consumo y la de los de producción:

14) $\mathrm{h} \equiv \mathrm{v} 2 / \mathrm{v} 1$

En la disputa con el capital, también se define la magnitud del trabajo excedente que, en calidad de plusvalor, va a favor de la empresa en cada sector. Dada la tasa salarial nominal (w) y los precios relativos en cada sector, esa masa de plusvalor extraído de los trabajadores se rea-

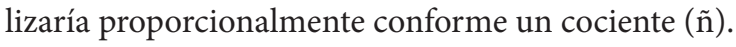

Nada garantiza que decisores autónomos y descentralizados encuentren en el mercado su exacta necesidad... y puedan pagarla. ${ }^{12}$ La realización de las tasas de ganancia, de explotación y composiciones orgánicas de capital individuales, emergen expost como parte de esas correspondencias contradictorias. La última fila de la tabla permite, además, apreciar las 
Tabla 1: Matriz de balances sectoriales (reproducción simple, dos sectores)

\begin{tabular}{|c|c|c|c|c|c|c|}
\hline \multirow{2}{*}{$\begin{array}{c}\text { BALANCES INTERSECTORIALES } \\
\text { SECTORES PRODUCTIVOS }\end{array}$} & \multicolumn{4}{|c|}{ FUENTES } & \multicolumn{2}{|c|}{ Usos } \\
\hline & $\begin{array}{c}\text { CAPITAL } \\
\text { CONSTANTE }\end{array}$ & VALOR & AÑADIDO & $\begin{array}{c}\text { PRODUCTO } \\
\text { SOCIAL }\end{array}$ & DEMANDA & SOCIAL \\
\hline PRODUCCIÓN DE MEDIOS DE PRODUCCIÓN & $\mathrm{cl}$ & v1 & s1 & p1 & $\mathrm{cl}$ & $\mathrm{c} 2$ \\
\hline PRODUCCIÓN DE MEDIOS DE CONSUMO & $\mathrm{c} 2$ & $\mathrm{v} 2$ & s2 & p2 & $\mathrm{v} 1+\mathrm{s} 1$ & $\mathrm{v} 2+\mathrm{s} 2$ \\
\hline ECONOMÍA & $\mathrm{C}$ & $\mathrm{V}$ & $\mathrm{s}$ & $\mathbf{P}$ & p1 & p2 \\
\hline CIRCULACIÓN & $\mathrm{D}$ & & G & $\mathbf{D}^{\prime}$ & d'1 & $d^{\prime} 2$ \\
\hline
\end{tabular}

Elaboración: autor.

determinaciones de la circulación sobre las capacidades de compra y realización de ese balance intersectorial problemático de principio entre valores de uso y de cambio.

El capital adelantado agregado fondea el potencial de compra de $\mathrm{C}$ - por tanto, reduciría la incertidumbre en el sector $\mathrm{I}-\mathrm{y}$ de $\mathrm{V}$, dejando en suspenso la realización de mercancías en el sector ir por el equivalente a $\mathrm{D}^{\prime}$ - D, pero ya estaría dinamizado el mercado del consumo capitalista por la circulación del poder de compra de las ganancias realizadas en la venta de C + $\mathrm{V}$, que a su vez alimentarían también, con la realización de ganancias en bienes de consumo suntuario, más compras de capitalistas, iterando tortuosamente por olas la evacuación de tramos de difícil demanda solvente, lo cual dificultaría la repetición del ciclo: el capital organiza un sistema monetario de producción que no puede continuar sin esa liquidez.

En valor de uso y valor de cambio se da un flujo de intercambios entre la producción de medios de producción y la producción de medios de consumo. Sin embargo, los ritmos, la orientación y la frontera de esos flujos pasan por la cristalización de una rentabilidad dada en el contexto no solo de un mercado solvente, sino, además, líquido. ${ }^{13}$

Los balances muestran un marco de desequilibrios, ${ }^{14}$ cuyos límites dependen de hasta dónde se tiene para comprar, basado en lo que se vendió -o lo que los actores relevantes converjan como posible venta, en caso de alguna opción de crédito, no contemplado aquí-. Esta circularidad, opuesta a la de la llamada Ley de Say de la economía convencional moderna y no solo por problemas de coordinación, sino de propiedad, distribución de las capacidades y los medios de pago y ganancias.

Al despejar términos algebraicos de las correspondencias inexorables en valor de cambio entre fuentes y usos de la tabla 1, aparece la identidad contable clave de la lógica macro que se impone sobre las decisiones y expectativas micro, porque lo que compra en neto el sector I al sector II tiene que financiarse con lo que le vende en neto:

15) $\mathrm{C} 2 \equiv \mathrm{v} 1+\mathrm{s} 1$

\section{ESTRUCTURA MATRICIAL DE LA LEY DEL VALOR, INTENCIONES MICRO Y RESULTADOS MACRO INESPERADOS}

La reproducción de la sociedad capitalista con un sujeto histórico escindido por el mercado depende de la validación social de los esfuerzos autónomos de infinidad de unidades productivas en una proporción que auspicie supervivencia en unos y ganancias aspiradas en otros. 
Estas proporcionalidades contradictorias determinan el paso siguiente de ascenso de lo abstracto a lo concreto a partir del análisis que Marx desarrolla en el volumen 1 de El capital, aunque nuestro autor no alcanzó a clarificar las complejas dinámicas sistémicas que emergen fruto de la forma específica de operación de la ley del valor a través del fetiche capitalista de los precios de producción. ${ }^{15}$

La matriz de proporcionalidades está definida por la operación de precios con ganancia en un sistema monetario de producción. ${ }^{16}$ El vector columna de valor añadido $(\mathrm{V}+\mathrm{S})$ se adjunta al de capital adelantado $(\mathrm{C})$ para formar una matriz de $2 \times 2$ cuyo determinante viene dado por:

16) $\operatorname{det}=\mathrm{c} 1(\mathrm{v} 2+\mathrm{s} 2)-\mathrm{c} 2(\mathrm{v} 1+\mathrm{s} 1)$

El determinante de la matriz que representa la reproducción simple con dos sectores sintetiza propiedades cruciales de la proporcionalidad en la sanción como valor de cambio de los requerimientos reproductivos que reclaman las relaciones técnicas entre trabajo vivo y trabajo muerto. ${ }^{17}$

La acumulación requiere mínimamente que el subespacio generado por los vectores se mantenga dentro del cuadrante de valores reales positivos. Mientras más cercanos a una ortogonalidad inalcanzable sean estos vectores, mayor será la magnitud del determinante, por tanto, mayor la latitud de las combinaciones en el ámbito de las proporciones viables entre trabajo muerto y trabajo vivo.

En cuanto el valor absoluto del determinante se acerque a cero, mayor será el vector fruto de la suma de los vectores de capital constante - trabajo muerto - y valor añadido — trabajo vivoque representa el total del producto social $(\mathrm{C}+\mathrm{V}+\mathrm{S}) \cdot{ }^{18}$ Otra condición emergente de la operación de la ley del valor que surge independiente de la voluntad de los capitales individuales.

Dada la identidad contable del balance intersectorial neto ineludible, el determinante de la reproducción simple queda definido por:

17) $\operatorname{det}=\mathrm{c} 1(\mathrm{v} 2+\mathrm{s} 2)-\mathrm{c}^{2}$

Entonces, el balance (SFC) entre sectores estructura esta expresión como los grados de libertad restantes cuando se descuenta el equivalente al cuadrado de las «importaciones mutuas» entre los dos sectores del área de transacciones posibles entre los requerimientos de capital constante comprados por los mismos productores de medios de producción y los requerimientos de medios de consumo comprados por su mismo sector productor. Expresando esta restricción macro emergente en términos de sus efectos sobre la agregación de las tasas de plusvalor y las composiciones orgánicas de capital en formación durante los procesos de producción y realización a nivel micro, queda claro que el sistema, más allá de la voluntad y condiciones técnicas de los actores individuales, impone que:

18) $d e t=\frac{o 1}{o 2} \frac{1+r 2}{1+r 1}-1$

Buena parte de los borroneos aritméticos intentados por Marx sin mucho éxito para ilustrar 
la perspicacia radical de su exploración encuentran nuevas luces en estos resultados algebraicos. Si la composición orgánica de capital es igual en ambos sectores, el determinante se define por la relación entre tasas de explotación:

19) det $=\frac{r 2-r 1}{1+r 1} \gtrless 0$ si $r 2 \gtrless r 1$

Si, en cambio, las tasas de explotación son iguales, es la relación entre composiciones orgánicas la que define la magnitud del determinante:

20) $\operatorname{det}=\frac{o 1}{o 2}-1 \gtrless 0$ si $01 \gtrless o 2$

Si ambas relaciones agregadas, composición orgánica y tasa de explotación, son iguales, los vectores columna no son linealmente independientes y el determinante es igual a cero. De similar modo, si se asume que las tasas de ganancia son iguales en ambos sectores, lo cual, además definiría que las tasas de plusvalor que finalmente se realizan en el mercado dependen unas de otras, independientemente de las condiciones técnicas, históricas y de lucha social locales:

$$
\text { 21) } r 1=r 2 \frac{(1+o 2)}{(1+o 1)}
$$

Otro ejemplo de las condiciones emergentes del agregado impuestas por la ley del valor a través del fetiche de la capitalización se deriva de los balances matriciales de la tabla 1, al sustituir las identidades contables netas intersectoriales en el producto total $(\mathrm{P})$ con el doble del monto del capital constante utilizado en la producción de medios de producción:

22) $\mathrm{P}=\mathrm{V} 2+\mathrm{s} 2+\mathrm{C} 1+2 \mathrm{c} 2=\mathrm{C}+\mathrm{D}_{2}(1+\mathrm{g} 2)$

Esto evidencia que aunque el impacto jerárquico de la producción de medios de producción en el plano ecológico del valor de uso es innegable, desde la perspectiva de la realización de las ganancias y la operación de los balances del valor de cambio, en la restricción vectorial que estamos estudiando tiene un peso dominante la dinámica del sector productor de bienes de consumo, ${ }^{19}$ fundamentando la prioridad macroeconómica desde Keynes en el desempeño de la demanda efectiva. ${ }^{20}$

A partir de esa restricción, la llamada «relación producto-capital» de la moderna discusión en torno al crecimiento (ICOR) ${ }^{21}$ puede evidenciarse también sometida a estas limitaciones sistémicas:

23) $\frac{P}{C}=1+\frac{c 2+v 2}{c 1+c 2}(1+g 2)=1+\frac{c 2(c 1+c 2)}{c 1 v 2+c 2^{2}}$ 
$\mathrm{Al}$ evidenciar la serie de determinaciones intersectoriales en la realización en valor de cambio de esta aproximación al ICOR, queda clara la trascendencia sistémica del balance neto y su dominancia sobre la forma valor que adoptan los efectos del progreso técnico sobre el producto social y sobre la relación entre trabajo necesario y excedente, aun si en la autopoiesis basamental ecológica se eleva el retorno insumo-producto en energía (EROI), presumiblemente factor fundamental de la evolución del ICOR deflactado.

A consecuencia del balance neto agregado entre sectores, la tasa de explotación en la producción de medios de producción (r1) necesariamente debe quedar constreñido por la composición orgánica de capital del sector y la proporción de capitales constantes sectoriales:

24) $r 1 \equiv(j o 1-1)$

Sin haber hipotizado comportamiento alguno y sin restricciones técnicas exante, emerge una nueva condición para la viabilidad de la acumulación capitalista que no aparece claramente en El capital.

Para que en el agregado del sector I pueda realizarse una masa de plusvalor positiva se requiere que la composición orgánica del capital en la producción de medios de producción sea estrictamente mayor que el cociente entre el capital constante invertido en el sector i y el ir:

25) $o 1>\left(\frac{1}{j}\right) \equiv\left(\frac{c 1}{c 2}\right)$

La tasa de explotación agregada del sector i también queda estructuralmente condicionada por su composición orgánica sectorial corregida por el cociente sectorial de capitales variables y plusvalores, reflejando las externalidades emergentes:

26) $r 2 \equiv\left(o 2-\frac{1}{h}\right) \tilde{\mathrm{n}}$

En correspondencia con la propiedad que emerge de este constreñimiento en la producción de medios de producción, tampoco podrán realizarse montos agregados positivos en el sector productor de bienes de consumo si su composición orgánica no rebasa el cociente entre el capital variable del sector en comparación al del otro, más allá de la pugna capital-trabajo al nivel de cada unidad productiva:

27) $02>\left(\frac{1}{h}\right) \equiv\left(\frac{v 1}{v 2}\right)$

Comprendiendo cómo queda condicionada la realización de la tasa de explotación en el agregado, estudiemos el comportamiento de las tasas de ganancia de cada sector como régimen continente de la realización de las tasas de ganancia individuales. ${ }^{22}$ En el Sector I sería:

28) $g 1 \equiv \frac{(j o 1-1)}{(o 1+1)}$

La primera derivada de la tasa de ganancia en la producción de medios de producción con 
respecto a la composición orgánica promedio es negativa y la segunda derivada es positiva, es decir, que se trata de una relación positiva aunque va haciéndose menos positiva a medida que crece la composición orgánica, sin nunca volverse negativa:
29) $\frac{\partial g 1}{\partial o 1} \equiv \frac{(j+1)}{(o 1+1)^{2}}>0$
30) $\frac{\partial^{2} g 1}{\partial o 1^{2}} \equiv \frac{-2(j+1)}{(o 1+1)^{3}}<0$

En el sector II también aparece restringida la rentabilidad por esta relación macro:

31) $g 2=\frac{(h o 2-1) \tilde{n}}{h(o 2+1)}$

El comportamiento de la tasa aparece ahora más complejo con respecto al cociente intersectorial entre capitales variables (h) y de montos de plusvalor (ñ), reflejando las externalidades que surgen de la agregación sistémica.

De manera similar, la primera derivada resulta positiva y la segunda, negativa:

32) $\frac{\partial g 2}{\partial o 2}=\frac{\left(\frac{o 2+1}{J}\right)-\left(\frac{o 2}{J}-1\right)}{(02+1)^{2}}=\frac{(j+1)}{j(o 2+1)^{2}}>0$

33) $\frac{\partial^{2} g 2}{\partial o 2^{2}}=\frac{-2(j+1)}{j(o 2+1)^{3}}<0$

El carácter de la relación entre la tasa de ganancia de cada sector y su composición orgánica a nivel de los agregados es relevante en torno al debate sobre la tendencia descendente de la tasa de ganancia. ${ }^{23}$ Aun si a nivel individual se logran resultados sostenidos en torno a la rentabilidad de la empresa, a nivel macro emergen presiones hacia la baja en la medida en que los incrementos proporcionales en valor de la relación trabajo pasado sobre trabajo vivo, que conduce la dinámica de la productividad del trabajo, no encuentra un ritmo similar en las mejoras proporcionales de la ganancia. ${ }^{24}$

\section{A MANERA DE CONCLUSIÓN}

La aplicación sistemática de la metodología de Consistencia Acervo-Flujo a las propiedades matriciales de la operación del fetiche del valor a través de precios de producción se muestra fructífera al tratar el más sencillo de los esquemas de reproducción de Marx: la reproducción simple con dos sectores, sin supuestos respecto al problema de la transformación de valores en precios, y su perfilamiento como propiedad emergente del sistema.

Fundamentalmente a partir de identidades contables, se ha podido derivar cómo, más allá de la intención micro exante, la maximización de la ganancia aparece entrampada en una serie de condicionamientos sistémicos y externalidades que surgen macroscópicamente.

A partir del balance neto intersectorial, el análisis de las vinculaciones endógenas entre vectores que expresan las relaciones agregadas orgánicas entre las tasas de explotación y ganancia 
con la composición orgánica del capital y los cocientes intersectoriales de capital constante, capital variable y plusvalor muestra el rol inexorable de la Consistencia Acervo-Flujo en la determinación de los macroagregados.

El ejercicio sugiere un campo de debate y profundización ineludible para el estudio ulterior y la transformación del subdesarrollo y las crisis.

\section{NOTAS}

1 Básicamente, en la parte editada y publicada por F. Engels como volumen II de El capital —parte 3, sobre todo, capítulos 20 y 21-, a partir de borradores no destinados a su publicación hasta el momento de la muerte del autor (cf. Marx, 1976 a y b). La subdivisión entre necesidades y lujos no es relevante en este trabajo ni se hacen supuestos particulares sobre la tasa de plusvalor o la composición orgánica del capital. Ver detalles de la ubicación de la temática en la rigurosa arquitectura que K. Marx había planificado para la exposición de su obra cumbre en Rosdolsky (2004), Harris (1972), Cockshott (2016), Gehrke (2018) y Echeverría (1994).

2 Ver, por ejemplo, desde diversas perspectivas las implicaciones de esta metodología, Lavoie (2014), Thirwall (2014), Sawyer y Veronese Passarella (2015). Básicamente, se establece que el déficit de unos actores necesariamente debe ser el superávit de otros y que, dadas las propiedades aditivas de los intercambios monetarios, inexorablemente esas condiciones se agregan y desagregan, congruentemente, por ramas y sectores. Estos flujos - ya de por sí históricamente determinados- generan acervos que a su vez interactúan con los flujos futuros. Para una antología reciente sobre la metodología con una introducción muy detallada de su aplicación a la Macroeconomía - por Alan Shipman — ver https:// academic.oup.com/cje/pages/sfc_macro_economics

3 El disciplinamiento y la proporcionalidad operados inconscientemente en los mercados perfilan las condiciones del funcionamiento «normal» del capitalismo que incluyen ciclos y crisis de diversa intensidad, profundidad y naturaleza. Ver, en diversas perspectivas, por ejemplo, Shaikh (1978), Cockshott (2016) o Bellofiore (2011).

4 Similar a lo asumido por K. Marx (1976a).

5 Esta reflexión ronda las búsquedas verbales de K. Marx tanto en el volumen II como en el III, sin que cuente entonces con las referencias paradigmáticas desarrolladas en las últimas décadas, incluso en medios académicos convencionales, dentro del paradigma de la complejidad. Ver Bellofiore (2011).

6 Ver Echeverría (1994). No es necesario aquí retomar la discusión sobre la transformación de valores en precios de producción, en tanto el punto es mostrar la intensa estructura implícita que la reproducciónrepetición - diferente- de los esfuerzos sociales dedicados a las correspondientes necesidades matriciales en valor de uso. Al respecto, hay valiosas convergencias en autores tan diversos como Cockshott (2016), Duménil y Foley (2008), Robinson (1951), Morishima (1973) o Harris (1972).

7 Cf. Bellofiore (2011), Shaikh (1978).

8 La suma de los valores deben ser iguales a la suma de los precios y la suma de la plusvalía debe equivaler a la suma de las ganancias - redistribuidas no solo por la tendencia a la perecuación de la tasa de ganancia, sino en un nivel más ascendente de concreción, por los contingentes factores de los precios de mercado- (cf. Marx, 1976a y b), Harris (1972), Echeverría (1994). Lastimosamente hay largos debates que pierden el punto resaltado en los resultados algebraicos que se desarrollan aquí, sin necesidad de penetrar en las visones neoricardianas o con un horizonte de equilibrio general, ajenos a la visión de Marx. Véase, por ejemplo, Robinson (1951), Morishima (1973), Harris (1972), Duménil y Foley (2008).

9 Cf. Marx (1974a). Ver también Cockshott (2016), que incluso permite en su página de internet acceder a un simulador en hoja electrónica https://docs.google.com/spreadsheets/d/1jSc1UxSFu9QBy7ojgRABe7zwRIc7_ o4cVqwBpnDtkw/edit\#gid=o). Asumiendo como dada fenoménicamente el 
nivel de ganancias, el análisis posterior se centra en las determinaciones macro que perfilan esos montos en los balances entre compras y ventas, como condición inexorable, independientemente de cualquier debate sobre la transformación de valores en precios.

10 Ver, por ejemplo, Cockshott (2016), Echeverría (1994).

11 Para detalles, ver la reflexión sobre la argumentación en K. Marx que lleva a representación que parecen simples, Echeverría (1994), Rosdolsky (2004).

12 Señalamiento constante en K. Marx luego recogido por otras corrientes del pensamiento como elemento crucial, por ejemplo, Sawyer y Veronese Passarella (2015).

13 Implicaciones explícitas en discusiones no enfocadas especialmente en temas monetarios como Echeverría (1994), Rosdolsky (2004), resaltada en su fuente en K. Marx por autores como Bellofiore (2011) y recogidas, sin mencionarlo, en la tradición keynesiana y poskeynesiana, por ejemplo, en Sawyer y Veronese Passarella (2015).

14 En contraste, por ejemplo, con cierta literatura muy abundante sobre el tema. Ver Morishima (1973), Harris (1972) Duménil y Foley (2008).

15 Ver, desde diversas comprehensiones, Rosdolsky (2004), Echeverría (1994), Cockshott (2016), Duménil y Foley (2008), Shaikh (1978).

16 Ver, por ejemplo, interpretaciones como las que relata Bellofiore (2011) en su trayectoria personal.

17 Las reflexiones respecto a la evolución de esa interdependencia como vector fundamental de la civilización y el rol específico del ser humano en el cosmos debería rastrearse hacia las elaboraciones de Leibniz en torno a la dialéctica entre fuerza viva y fuerza muerta.

18 Un análisis más detallado del determinante en el contexto de los esquemas de reproducción rebasa el alcance de este artículo, pero puede demostrarse, entre otras implicaciones, su signo depende de la diferencia ponderada - por la composición orgánica referida al valor añadido- de las tasas de plusvalor entre los sectores: [o'1 $(1+\mathrm{r} 2)-(1+\mathrm{r} 1)]$.

19 Ver Echeverría (1994).

20 Cf. Lavoie (2014).

21 En una perspectiva marxista hay toda una tradición de tratamiento de relaciones productivistas que liguen asignación en distintas medidas de valor de uso y de cambio con sus resultados, como la relatada en Shaikh (1978).

22 Factor generalmente no considerado en el debate de la transformación de valores en precios, desde diversas perspectivas, ver, por ejemplo Duménil y Foley (2008), Harris (1972), Morishima (1973) o Bellofiore (2011).

23 Elemento tampoco presente en los debates al respecto, por ejemplo, Bellofiore (2011), Duménil y Foley (2008), Harris (1972) y Shaikh (1978).

24 Similares razonamientos, sin el soporte algebraico, se deducen en Echeverría (1994).

\section{REFERENCIAS}

Bellofiore, R. (2011). Crisis theory and the Great Recession: a personal journey, from Marx to Minsky. In Revitalizing Marxist Theory for Today's Capitalism (pp. 81-120). Emerald Group Publishing Limited.

Cockshott, W. P. (2016). Marxian reproduction prices versus prices of production: probability and convergence. (s. d.).

Duménil, G. and Foley, D. (2008). The Marxian transformation problem. (s. d.). The New Palgrave: A Dictionary of Economics, Palgrave Macmillan, Basingstoke.

Durlauf, S. N. and Blume, L. (Eds.). (2008). The new Palgrave dictionary of economics (vol. 6). Basingstoke, uk: Palgrave Macmillan.

Echeverría, B. (1994). Circulación capitalista y reproducción de la riqueza social. Quito, Ecuador: Nariz del Diablo. 
Gehrke, C. (2018). Marx's reproduction schemes and multi-sector growth models. The European Journal of the History of Economic Thought. doi: 10.1080/09672567.2018.1475500

Harris, D. J. (1972). On Marx's scheme of reproduction and accumulation. Journal of Political Economy, $80(3), 505-522$.

Lavoie, M. (2014). Post-Keynesian economics: new foundations. Cheltenham, uk: Edward Elgar Publishing.

Marx, K. (1976a). El capital, t. II, vol 4. México DF, México: Ed. Siglo xxı.

Marx, K. (1976b). El capital, t. II, vol 5. México DF, México: Ed. Siglo xxI.

Marx, K. (1978). El capital, t. III, vol 7. México DF, México: Ed. Siglo xxi.

Morishima, M. (1973). Marx's Economics a dual theory of value and growth. Cambridge, uK: University Press.

Robinson, J. (1951). «Introduction» to Rosa Luxemburg. The Accumulation of Capital. New York, USA: (s. d.).

Rosdolsky, R. (1978). Génesis y estructura de El capital de Marx. México DF, México: Siglo xxI.

Sawyer, M. and Veronese Passarella, M. (2017). The monetary circuit in the age of financialisation: a stock-flow consistent model with a twofold banking sector. Metroeconomica, 68(2). doi: 10.1111/ meca.12103.

Shaikh, A. (1978). An introduction to the history of crisis theories. US capitalism in crisis, (pp. 219-241). Editorial Collective Union for Radical Political Economics.

Thirlwall, A. P. (2014). The balance of payments constraint as an explanation of the international growth rate differences. PSL Quarterly Review, 45-53. 
